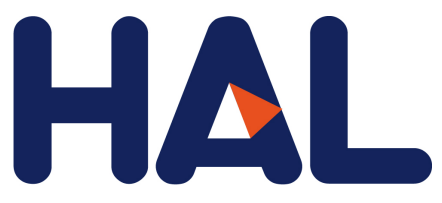

archives-ouvertes

\title{
Engineering the emission of light from a scanning tunneling microscope using the plasmonic modes of a nanoparticle
}

Eric Le Moal, Sylvie Marguet, Damien Canneson, Benoît Rogez, Elizabeth Boer-Duchemin, Gérald Dujardin, T.V. Teperik, Dana-Codruta Marinica, Andrey G. Borisov

\section{To cite this version:}

Eric Le Moal, Sylvie Marguet, Damien Canneson, Benoît Rogez, Elizabeth Boer-Duchemin, et al.. Engineering the emission of light from a scanning tunneling microscope using the plasmonic modes of a nanoparticle. Physical Review B: Condensed matter and materials physics, American Physical Society, 2016, 93, pp.035418. 10.1103/PhysRevB.93.035418 . cea-01257655

HAL Id: cea-01257655

https://hal-cea.archives-ouvertes.fr/cea-01257655

Submitted on 18 Jan 2016

HAL is a multi-disciplinary open access archive for the deposit and dissemination of scientific research documents, whether they are published or not. The documents may come from teaching and research institutions in France or abroad, or from public or private research centers.
L'archive ouverte pluridisciplinaire HAL, est destinée au dépôt et à la diffusion de documents scientifiques de niveau recherche, publiés ou non, émanant des établissements d'enseignement et de recherche français ou étrangers, des laboratoires publics ou privés. 


\title{
Engineering the emission of light from a scanning tunneling microscope using the plasmonic modes of a nanoparticle
}

\author{
Eric Le Moal, ${ }^{1, *}$ Sylvie Marguet, ${ }^{2}$ Damien Canneson, ${ }^{1}$ Benoît Rogez, ${ }^{1}$ Elizabeth Boer-Duchemin,,${ }^{1}$ Gérald Dujardin, ${ }^{1}$ \\ Tatiana V. Teperik, ${ }^{3}$ Dana-Codruta Marinica, ${ }^{1}$ and Andrey G. Borisov ${ }^{1, \dagger}$ \\ ${ }^{1}$ Institut des Sciences Moléculaires d'Orsay (ISMO), CNRS, Univ Paris Sud, Université Paris-Saclay, F-91405 Orsay, France \\ ${ }^{2}$ NIMBE, CEA, CNRS, Université Paris-Saclay, CEA Saclay 91191 Gif-sur-Yvette, France \\ ${ }^{3}$ Donostia International Physics Center DIPC, Paseo Manuel de Lardizabal 4, 20018, Donostia-San Sebastían, Spain
}

(Received 24 September 2015; revised manuscript received 3 December 2015; published 11 January 2016)

\begin{abstract}
The inelastic tunnel current in the junction formed between the tip of a scanning tunneling microscope (STM) and the sample can electrically generate optical signals. This phenomenon is potentially of great importance for nano-optoelectronic devices. In practice, however, the properties of the emitted light are difficult to control because of the strong influence of the STM tip. In this work, we show both theoretically and experimentally that the sought-after, well-controlled emission of light from an STM tunnel junction may be achieved using a nonplasmonic STM tip and a plasmonic nanoparticle on a transparent substrate. We demonstrate that the native plasmon modes of the nanoparticle may be used to engineer the light emitted in the substrate. Both the angular distribution and intensity of the emitted light may be varied in a predictable way by choosing the excitation position of the STM tip on the particle.
\end{abstract}

DOI: 10.1103/PhysRevB.93.035418

\section{INTRODUCTION}

Electrically driven nanosources of light will play a key role in the development of devices integrating both nanoelectronics and nanophotonics, one of today's main challenges in electronics [1-3]. A nanoscale tunnel junction provides the simplest way to convert low-energy electrons into photons with the smallest of footprints as compared to other nanosources of light such as nanoscale light emitting diodes [3] and semiconductor nanolasers [4]. Until recently [5,6], much less attention has been paid to nanosources of light based on a nanoscale tunnel junction [7]. These recent studies confirm the rapidly growing interest in tunnel junctions as key elements for the design of electrically driven nanosources of light with controllable emission.

Photon emission from a tunnel junction is associated with inelastic electron tunneling (IET) when electrons undergo a transition from higher to lower energy levels and transfer part of their energy to photons [8-12]. When possible, excitation of the plasmon modes in the junction resonantly enhances the intensity of the emitted light $[9,11,12]$. Moreover, the plasmonic response of optical nanoantennas coupled to a tunnel junction has been recently used to control the emitted light $[5,6]$. This IET effect has been widely studied, especially since the invention of the scanning tunneling microscope (STM) [11-25] (see also Ref. [26] and references therein). However, using metallic nanostructures with tunneling gaps as nanosources of light has been hampered by a lack of control of the emission properties. In most experiments, the emitted light is completely dominated by the resonance of the gap plasmon mode induced by the strong tip-sample interaction when both the tip and the sample are made of plasmonic materials such as gold or silver [22,25,27]. The

\footnotetext{
*eric.le-moal@u-psud.fr

†andrei.borissov@u-psud.fr
}

extreme spatial confinement of the gap plasmon inside the junction makes it exceedingly sensitive to very slight yet unavoidable and unpredictable changes in the morphology of the tip apex $[28,29]$. Consequently, the common wisdom in the field is that STM-induced light emission suffers from this "arbitrary" character [29]. Similar issues have also been reported for plasmonic nanostructures on a plasmonic (or highly-reflective) substrate, where a gap plasmon occurs at the nanostructure-substrate interface [30,31].

Nonetheless, a recent experiment using a tip made of a nonplasmonic material (i.e., tungsten) and a transparent substrate (i.e., glass) suggests that the STM-induced light emitted from a gold nanoparticle (NP) may be controlled in a reproducible way by changing the excitation position of the tip on the NP [32]. Understanding and mastering this effect opens up new possibilities for engineering the light emitted from an STM tunnel junction by using the resonant modes of a plasmonic nanostructure. Here, we propose a new theoretical framework within which the emission properties of the tip-NP system may be predicted. Experimental data (taken in part from Ref. [32]) is used to test this approach.

In this paper we demonstrate that the properties of the light emitted from an STM tip-plasmonic NP junction may be predicted and controlled in a simple way. For a sufficiently small radius of curvature as compared to the NP size, the nonplasmonic tip need not to be treated explicitly. Essentially, the effect of the tip results in the renormalization of the fluctuating (point) dipole associated in classical descriptions with inelastic tunneling current $[11,12]$. The dependence of the angular profile and intensity of the emitted light as a function of the tip position may be obtained from the Green's tensor $\mathbb{G}_{\mathrm{NP}}$ of the plasmonic NP only [33-35]. In particular, simple and intuitive insights may be gained by considering the development of $\mathbb{G}_{\mathrm{NP}}$ in terms of the localized surface plasmon (LSP) eigenmodes [36-38]. We validate our approach by comparing experimental data with the results of electromagnetic calculations performed using complete and simplified representations of the tip-NP system. 
(a)

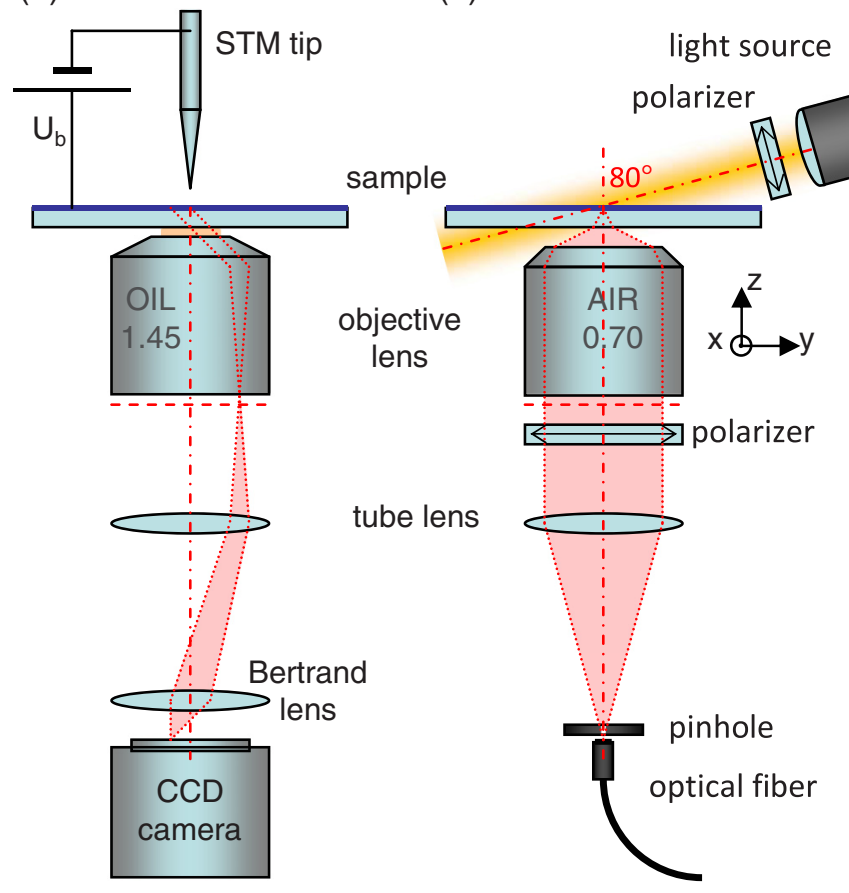

FIG. 1. Experimental setups. (a) Fourier imaging of the STMinduced light emitted from metallic nanostructures on a transparent substrate. (b) Darkfield optical spectroscopy of a single nanostructure upon illumation with polarized white light in grazing incidence. In both setups, light is collected through the substrate. Inscriptions on the housing of the objective lenses indicate the immersion medium and numerical aperture.

\section{EXPERIMENTAL METHODS}

Figure 1(a) shows a sketch of the experimental setup used for the study of the STM-induced light emission from gold NPs. The setup consists of a commercial STM/AFM head operating in air in ambient conditions, mounted on top of an inverted optical microscope [39]. A nanopositioning stage maintains the sample between the STM tip and the objective lens. The sample is biased to $2.8 \mathrm{~V}$, while the tip is grounded. We use tungsten tips made by electrochemical etching in sodium hydroxide, which typically have curvature radius of $30 \mathrm{~nm}$ at the apex. The light emitted in the substrate is collected using a high numerical aperture objective lens (oil immersion, $\mathrm{NA}=1.45$ ). The angular distribution of the emitted light is retrieved by optical imaging in the Fourier plane using a water-cooled CCD camera, with the tip located at a fixed position on the sample.

A different setup is used to measure the light scattering spectra of the gold NPs [see Fig. 1(b)]. The sample is illuminated with a collimated beam of polarized white light at grazing incidence $\left(\approx 80^{\circ}\right.$ off the $z$ axis). The light scattered in the substrate is collected using an air objective lens of sufficiently low NA to reject the incident light $(\mathrm{NA}=0.7$, angle of acceptance $\approx 44^{\circ}$ ). The polarization of the incident light is controlled using a polarizer in front of the light source. The collected light is focused on the end of an optical fiber bundle that is coupled to an optical spectrometer. A pinhole is placed just before the fiber end in order to select the light
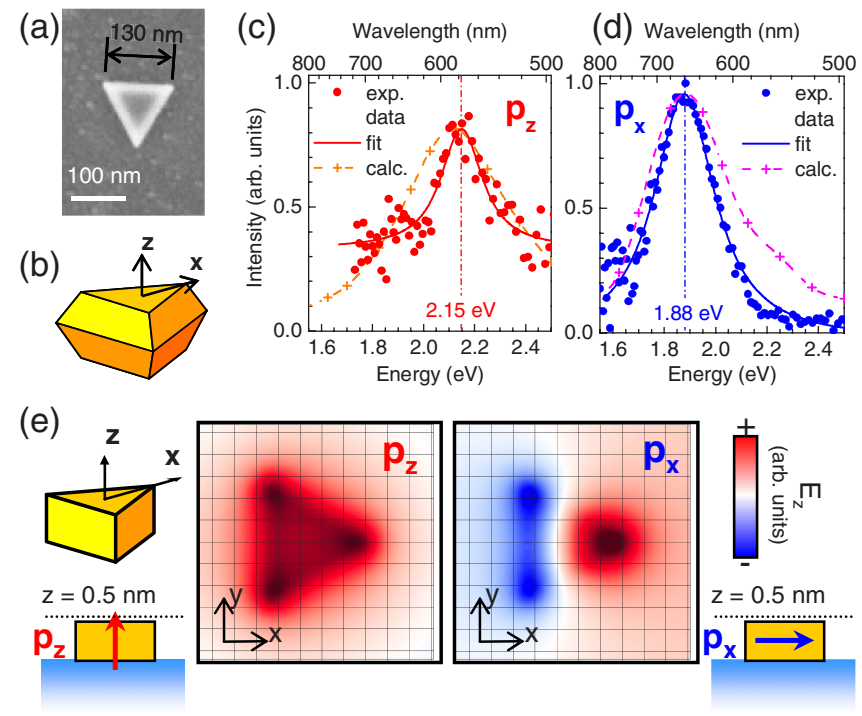

FIG. 2. Characterization of an individual plasmonic NP. (a) Scanning electron micrograph of a gold nanotriangle and (b) sketch of its truncated bitetrahedral shape. (c) and (d) Calculated (crosses and dashed lines) and experimental (circles) light scattering spectra for vertical $\left(\mathbf{p}_{\mathbf{z}}\right)$ and in-plane $\left(\mathbf{p}_{\mathbf{x}}\right)$ incident light polarization. Experimental data are obtained by dark-field optical microscopy upon polarized white light illumination of an individual NP. Experimentally observed resonances are fitted with single Lorentzian profiles yielding $2.15 \mathrm{eV}$, and $1.88 \mathrm{eV}$ for the vertical $\left(\mathbf{p}_{\mathbf{z}}\right)$ and in-plane $\left(\mathbf{p}_{\mathbf{x}}\right)$ dipolar LSP resonances, respectively. (e) Theoretical distribution of the vertical component of the electric field $\left(E_{z}\right)$ in the horizontal $(x, y)$ plane $0.5 \mathrm{~nm}$ above the NP. Results are shown for the excitation of the $p_{z}$ and $p_{x}$ dipolar LSPs. (Inset) Sketch of the simplified NP geometry used in the $E_{z}$ calculations.

originating from a $2-\mu \mathrm{m}$ area of the sample plane. Thus the spectrum of the light scattered by a single gold NP can be measured. When $s$-polarized light is used to illuminate the sample, only the in-plane modes (along the $x$ axis) of the NP are excited, whereas a combination of vertical $(z$ axis) and in-plane ( $y$ axis) modes is excited with $p$-polarized light, since the incidence angle is not $90^{\circ}$. In the latter case, a second polarizer is thus added between the objective and tube lenses, with its transmission axis along the $x$ axis, so that the contribution from the in-plane modes is rejected. In this way, we disentangle the contributions of the two types of modes. Note that the resonances of the in-plane and vertical dipolar modes of the NPs were wrongly assigned in Fig. S3 of Ref. [32], because such a two-polarizer scheme was not used.

Figure 2(a) shows a scanning electron micrograph of a gold NP typical of those used in this study. These are gold nanotriangles with a truncated bitetrahedral shape, which are $128 \pm 6 \mathrm{~nm}$ in edge length and $69 \pm 7 \mathrm{~nm}$ in thickness. The NPs are deposited on a glass coverslip coated with an optically thin indium-tin-oxide layer (ITO, $100 \mathrm{~nm}$ ).

\section{RESULTS AND DISCUSSION}

The plasmon response of the individual gold NPs deposited on the ITO-coated glass substrate (the STM tip is not present at this stage) is characterized in Fig. 2. This figure 
shows experimentally and using a complete electromagnetic calculation, that two distinct dipolar modes exist in the NPs studied. Darkfield spectroscopy of individual NPs obtained using $p$ and $s$-polarized light [see setup in Fig. 1(b)] reveals strong scattering resonances at $2.1 \pm 0.1$ and $1.9 \pm 0.1 \mathrm{eV}$, as shown in Figs. 2(c) and 2(d).

The experimentally measured scattering resonances are retrieved using a rigorous solution of the Maxwell equations based on the pseudospectral Lanczos wave packet propagation method [40]. The dipole induced at the NP in response to an incident plane wave is calculated using the dielectric function of gold from Ref. [41] and the realistic shape of the NP as sketched in Fig. 2(b).

The electric nearfield distributions in a plane just above the $\mathrm{NP}$, calculated at the two resonance frequencies, are shown in Fig. 2(e). One can unambiguously assign the observed resonances to the vertical $\left(\mathbf{p}_{\mathbf{z}}, 2.1 \mathrm{eV}\right)$ and in-plane $\left(\mathbf{p}_{\mathbf{x}}, 1.9 \mathrm{eV}\right)$ dipolar LSP modes. As expected for dipolar modes, the vertical $p_{z}$ resonance at $2.1 \mathrm{eV}$ with an induced dipole oriented along the $z$ axis is associated with a relatively flat distribution of the $E_{z}$ component of the electric near field over the upper NP face, except for amplitude peaks at the vertices. The $E_{z}$ field component of the in-plane $p_{x}$ resonance at $1.9 \mathrm{eV}$ with an induced dipole oriented along the $x$ axis exhibits a nodal structure with sign reversal on the center of the upper face. It is these electric near-field distributions that will be used in one of the approaches proposed below to predict the intensity and angular distribution of the emitted light as a function of the tip excitation position on the NP. Due to the small NP size, we may restrict ourselves to the dipolar resonances since higher-order multipole resonances [42-44] are comparatively much less pronounced, and occur above $2.4 \mathrm{eV}$ according to our calculations.

Because of the multiscale character of the problem addressed in this study, the computations are numerically demanding. Indeed, the nanometer-sized junction between the tip and the surface must be well resolved. The nano-object with a characteristic size of $\sim 100 \mathrm{~nm}$ must also be described. As well, the size of the computational box must be at the wavelength scale $(\sim 1000 \mathrm{~nm})$ so that the near fields are not distorted. In order to reduce the computational effort and the meshing complexity we performed most of the calculations in this study [except those in Figs. 2(c) and 2(d)] using a simple Drude model for the dielectric functions, and the NP geometry approximated as a triangular nanoprism with right-angled side faces as sketched in Fig. 2(e). For consistency with the rest of the theoretical results in this paper, the near fields in Fig. 2(e) are obtained using these approximations.

Inelastic electron tunneling (IET) in the junction between the tungsten STM tip and gold NP leads to light emission [32] [see setup in Fig. 1(a)]. As shown in Fig. 3, for a tip excitation position close to the vertex of the triangle, the intensity distribution in the Fourier plane measured below the substrate reveals that the emitted light is primarily directed away from the particle. Because of the small NP size, the general emission pattern may be approximated by that of a tilted electric dipole $\mathbf{P}$ on an air/glass interface [32] with a tilt angle $\Theta=\arctan \left(P_{\|} / P_{z}\right)$ measured with respect to the $z$ axis (i.e., the STM tip axis). Here, $P_{\|}$and $P_{z}$ are the "effective" dipole moment components in the $(x, y)$ plane (parallel to the

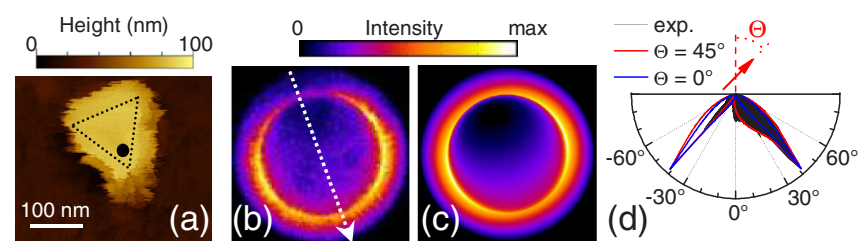

FIG. 3. (a) STM topography image of a single gold nanotriangle on ITO. (b) Measured Fourier plane emission pattern from this gold nanotriangle upon electrical excitation with the tungsten tip located at the position indicated in panel (a), $I_{\text {tunnel }}=0.9 \mathrm{nA}$, sample bias $U_{b}=2.8 \mathrm{~V}$. (c) Calculated Fourier-plane image. Here, we consider analytically a radiating point electric dipole on an air-glass interface [45]. For this particular excitation position, the dipole tilt angle with respect to the tip-axis is determined to be $\Theta=45^{\circ}$. (d) Polar plot of intensity profiles taken from the experimental and theoretical Fourierplane images along the white dotted arrow in (b). The analytic dipole model result for $\Theta=0^{\circ}$ is also shown.

NP surface) and along the $z$ axis, respectively. For the data in Fig. 3, the best fit is obtained assuming this "effective" dipole oriented at $\Theta \approx 45^{\circ}$. Slight discrepancies between the experimental and calculated Fourier images are presumably due to the contributions of higher multipole moments of the NP-tip system. In the following, we use this parameter $\Theta$ to compare the experimental and calculated results. In the experiment, $\Theta$ represents the orientation of the "effective" tilted dipole which best fits the Fourier plane angular emission results, and in the calculations $\Theta$ is equivalent to the orientation of the induced dipole in the NP (or in the NP-tip system).

Before further discussion of the results, let us outline theoretical considerations that provide a general framework for understanding the data. Due to nanometer size of the junction, we use a classical picture where the inelastic tunneling current is represented as a point electric dipole p placed in the junction and radiating at the transition frequency $\omega$. The dipole amplitude is given by the transition matrix elements $[13,15-18,20,22-25,46]$ or, according to recent theory, by the corresponding spectral components of the quantum noise [47]. This dipole $\mathbf{p}$ is located above the NP surface at position $\mathbf{r}_{\mathbf{d}}$ which is typically set at the middle of the junction, and is oriented along the direction of the tunneling current, i.e., parallel to the tip axis ( $z$ axis) when the tip is on the horizontal upper NP face. The total radiated electric field is given by

$$
\mathbf{E}_{\text {tot }}=\mathbf{E}_{\mathrm{d}}+\mathbf{E}_{\mathrm{NP}}^{+}+\mathbf{E}_{\mathrm{T}}^{+},
$$

where $\mathbf{E}_{\mathbf{d}}$ is the field of the point dipole $\mathbf{p}$ and

$$
\mathbf{E}_{\mathrm{NP} / \mathrm{T}}^{+}=\mathcal{R}_{\mathrm{NP} / \mathrm{T}}\left(\mathbf{E}_{\mathbf{d}}+\mathbf{E}_{\mathrm{T} / \mathrm{NP}}^{+}\right),
$$

is the field scattered by the NP or tip (T). Here, $\mathcal{R}_{\mathrm{NP} / \mathrm{T}}$ is the operator that connects the incident and scattered fields for the $\mathrm{NP} / \mathrm{T}$. Obviously, $\mathcal{R}_{\mathrm{NP} / \mathrm{T}}$ is directly linked with the NP or tip Green's dyadic $\mathbb{G}_{\mathrm{NP} / \mathrm{T}}[48,49]$ as follows from analysis of the fields created by the point dipole excitation of the individual 
NP or tip:

$$
\begin{aligned}
\mathbf{e}_{\mathrm{NP} / \mathrm{T}} & =\mathcal{R}_{\mathrm{NP} / \mathrm{T}} \mathbf{E}_{\mathbf{d}}+\mathbf{E}_{\mathbf{d}}=\left(\mathcal{R}_{\mathrm{NP} / \mathrm{T}}+1\right)\left(-4 \pi \frac{\omega^{2}}{c^{2}} \mathbb{G}_{0}\right) \mathbf{p} \\
& =\left(-4 \pi \frac{\omega^{2}}{c^{2}} \mathbb{G}_{\mathrm{NP} / \mathrm{T}}\right) \mathbf{p},
\end{aligned}
$$

where $\mathbb{G}_{0}$ is the free space dyadic, and the field of the point dipole is given by $\mathbf{E}_{\mathbf{d}}=-4 \pi \frac{\omega^{2}}{c^{2}} \mathbb{G}_{0} \mathbf{p}$. From Eq. (3), the Green's dyadic of the NP or tip can be expressed as

$$
\mathbb{G}_{\mathrm{NP} / \mathrm{T}}=\left(1+\mathcal{R}_{\mathrm{NP} / \mathrm{T}}\right) \mathbb{G}_{0} .
$$

From Eq. (2), one obtains the field scattered by NP and STM tip:

$$
\mathbf{E}_{\mathrm{NP}}^{+}=\left[\frac{\mathcal{R}_{\mathrm{NP}} \mathcal{R}_{\mathrm{T}}}{1-\mathcal{R}_{\mathrm{NP}} \mathcal{R}_{\mathrm{T}}}\left(1+\mathcal{R}_{\mathrm{NP}}\right)+\mathcal{R}_{\mathrm{NP}}\right] \mathbf{E}_{\mathbf{d}} .
$$

This and a similar expression for $\mathbf{E}_{\mathrm{T}}^{+}$can be substituted into Eq. (1) to obtain the sought-after electric field. Note that $\mathcal{R}_{\mathrm{NP}} \mathbf{E}_{\mathbf{d}}$ is the field created by the NP in response to the point dipole excitation.

The description may be greatly simplified using the assumption that for the present system the light emission is dominated by the NP contribution due to the antenna effect [50]. Indeed, only the gold NP responds resonantly to the excitation. The real part of the dielectric permittivity of tungsten is positive for the investigated $\omega$ range, i.e., the tip is not plasmonic [51,52]. Thus, the emitted radiation is approximately given by $\mathbf{E}_{\text {tot }}=\mathbf{E}_{\mathrm{NP}}^{+}+\mathbf{E}_{\mathbf{d}}$. The validity of this approximation is demonstrated below by the direct calculation of the NP and tip contribution to the total polarization of the system. Thus we can write

$$
\begin{aligned}
\mathbf{E}_{\mathbf{t o t}} & =\mathbb{S}\left(1+\mathcal{R}_{\mathrm{NP}}\right)\left(-4 \pi \frac{\omega^{2}}{c^{2}} \mathbb{G}_{0}\right) \mathbf{p} \\
& =\mathbb{S}\left(-4 \pi \frac{\omega^{2}}{c^{2}} \mathbb{G}_{\mathrm{NP}}\right) \mathbf{p},
\end{aligned}
$$

where $\mathbb{S}$ is given by

$$
\mathbb{S}=\left(\frac{\mathcal{R}_{\mathrm{NP}} \mathcal{R}_{\mathrm{T}}}{1-\mathcal{R}_{\mathrm{NP}} \mathcal{R}_{\mathrm{T}}}+1\right) .
$$

In the nonretarded limit, $\mathbb{S}$ expresses the effect of the multiple electrostatic images of the point dipole $\mathbf{p}$ created by the coupled NP-tip system.

Considering the nanometer-scale junction between the NP surface and the tip, as well as the tip curvature radius $(\approx 30 \mathrm{~nm})$ which is small compared to the NP size $(\approx 130 \mathrm{~nm}$ in edge length), the enhanced near fields are confined to a localized spot. Therefore, as a reasonable approximation, the operator $\mathbb{S}$ can be replaced by the scalar value $S$ so that

$$
\mathbf{E}_{\text {tot }}=-4 \pi \frac{\omega^{2}}{c^{2}} \mathbb{G}_{\mathrm{NP}} \tilde{\mathbf{p}},
$$

where $\tilde{\mathbf{p}}=S \mathbf{p}$. Thus the field emitted from the STM junction may be interpreted as the field resulting from the excitation of the NP by the effective point dipole $\tilde{\mathbf{p}}$ given by the simple rescaling of the inelastic tunneling dipole $\mathbf{p}$.

Consistent with the small radius of curvature and the nonplasmonic character of the $\mathrm{W}$ tip, our calculations do not show any spectral features in addition to NP resonances. Furthermore, the (broad) NP plasmon resonances are only slightly perturbed by the $\mathrm{W}$ tip. Indeed, we measure and calculate relatively small $(<0.2 \mathrm{eV})$ red shifts of the resonances in the presence of the STM tip located $1 \mathrm{~nm}$ above the NP surface. The $\omega$ dependence of $\tilde{\mathbf{p}}$ is determined by the IET power spectrum [11,14,53], and Eq. (8) describes the field created by a point dipole located at $\mathbf{r}_{\mathbf{d}}$ above the NP surface and oriented along the tip axis $\tilde{\mathbf{p}}=\hat{\mathbf{e}}_{\mathbf{z}} \tilde{p}$. We use $\hat{\mathbf{e}}_{i}(i=x, y, z)$ to denote the unit length vector along the corresponding axis. In our calculations, we use $\mathbf{r}_{\mathbf{d}}$ given by the coordinates of the middle of the junction but explicit tests have shown that the results are robust with respect to $\mathrm{nm}$ variations in this dipole position. Thus the effect of the tip location on the intensity and angular profile of the emitted light may be predicted from the Green's function of the NP only since $\mathbf{E}_{\text {tot }} \propto \mathbb{G}_{N P} \hat{\mathbf{e}}_{\mathbf{z}}$. The Green's function $\mathbb{G}_{\mathrm{NP}}$ expansion in terms of the plasmonic eigenmodes of the NP [36-38] leads to

$$
\mathbf{E}_{\mathbf{t o t}} \approx \sum_{n} \frac{4 \pi \omega^{2}}{\omega^{2}+i \omega \eta_{\mathrm{n}}-\omega_{\mathrm{n}}^{2}}\left(\hat{\mathbf{e}}_{\mathbf{z}} \mathbf{f}_{\mathrm{n}}^{*}\left(\mathbf{r}_{\mathbf{d}}\right)\right) \mathbf{f}_{\mathrm{n}}(\mathbf{r}),
$$

where $\mathbf{f}_{\mathrm{n}}(\mathbf{r}), \omega_{\mathrm{n}}$, and $\eta_{\mathrm{n}}$ represent, respectively, the field, frequency, and attenuation of the $n$th plasmonic eigenmode of the NP.

If only the dipolarlike modes are retained because of the small NP size, the fields $\mathbf{f}_{\mathrm{n}}$ are those created by the $n$th plasmon eigendipole $\mathbf{p}_{\mathrm{n}} \cdot \mathbf{p}_{\mathrm{n}}$ can be obtained by integrating over the NP volume the polarization density vector corresponding to the $n$th plasmonic eigenmode. For the broad overlapping resonances here, the $\omega$ dependence of the expansion coefficients may be neglected. Then, direct comparison between $\mathbf{E}_{\text {tot }}$ given by Eq. (9) and the field radiated by the ensemble of the point dipoles allows to conclude that Eq. (9) describes the electric field radiated by the induced dipole at the NP:

$$
\mathbf{P}=\mathcal{C} \sum_{n}\left[\hat{\mathbf{e}}_{\mathbf{z}} \mathbf{f}_{\mathrm{n}}^{*}\left(\mathbf{r}_{\mathbf{d}}\right)\right] \mathbf{p}_{\mathrm{n}},
$$

where $\mathcal{C}$ is a constant, and $\hat{\mathbf{e}}_{\mathbf{z}} \mathbf{f}_{\mathrm{n}}^{*}$ is essentially the $E_{z}$ map of plasmonic eigenfields [as shown in Fig. 2(e)]. The nanotriangle supports four basic dipolar excitations: a vertical dipole perpendicular to the substrate, and three degenerate in-plane dipolar modes [42,43], which can be expressed as $\mathbf{p}_{\mathbf{x}}=\hat{\mathbf{e}}_{\mathbf{x}} p_{\|}$, and $\mathbf{p}_{ \pm}=\left[-\frac{1}{2} \hat{\mathbf{e}}_{\mathbf{x}} \pm \frac{\sqrt{3}}{2} \hat{\mathbf{e}}_{\mathbf{y}}\right] p_{\|}$.

In order to test the validity of the models of Eqs. (8) and (10), we compare the experimental data with theoretical results of decreasing level of complexity: (i) rigorous calculations including the complete NP-tip coupled system [40]; (ii) model calculations based on Eq. (8) where the NP has been excited with a point dipole. Here, scattering by the tip and the $\omega$ dependence of $\tilde{\mathbf{p}}$ are neglected, but the Green's function of the NP $\mathbb{G}_{N P}$ is calculated exactly using the COMSOL MULTIPHYSICS package; ${ }^{1}$ (iii) model calculations based on $E_{z}$ maps of the plasmonic eigenfields, as given by Eq. (10) where the $\mathbf{f}_{\mathrm{n}}$ fields of the NP plasmon eigenmodes are calculated at the resonance frequency, i.e., only the resonant dipolar contributions are taken into account. This makes the calculations in (iii) much

${ }^{1}$ COMSOL MULTIPHYSICS package, version 4.2a, www.comsol.com 
(a)

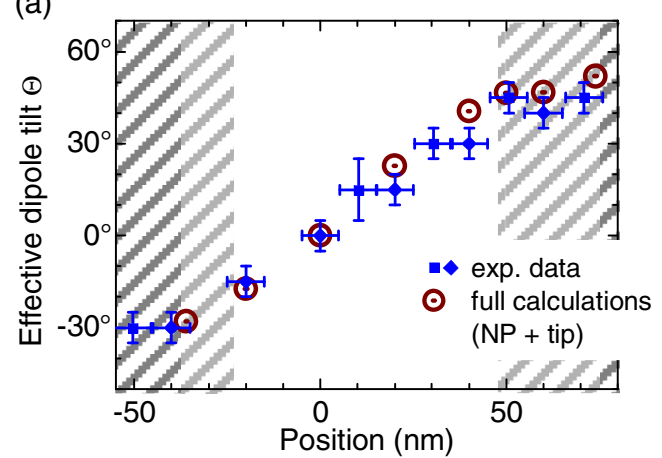

(b)

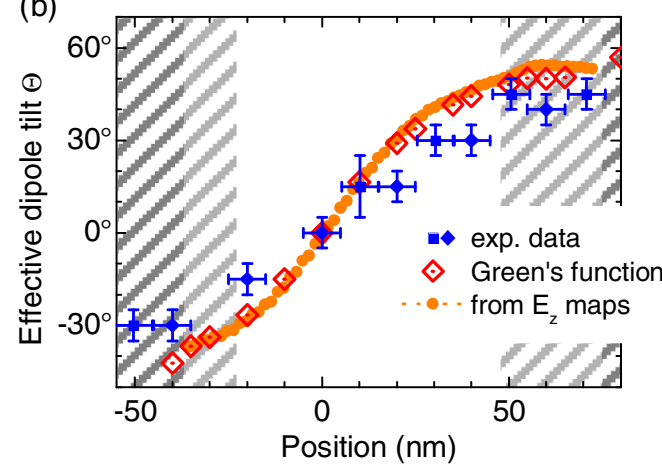

(c)

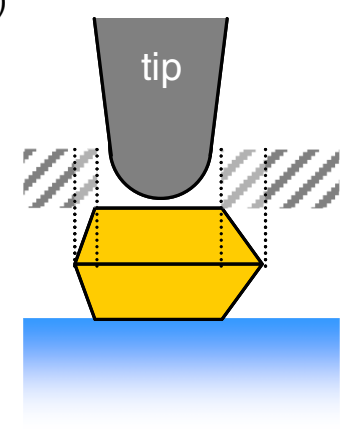

(d)

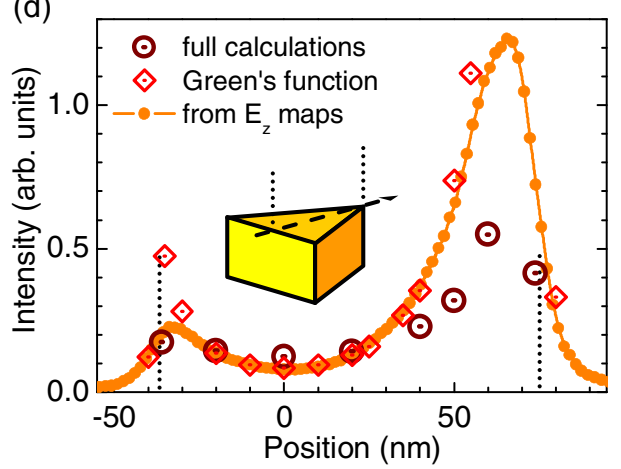

FIG. 4. Comparison of experiment and theory: emission properties of an STM-excited gold NP. (a) and (b) Tilt angle $\Theta$ of the "effective emission dipole." (c) Schematic of the NP-tip system, showing the truncated bitetrahedral shape of the NP. Shaded areas indicate tip positions where the tip apex is above a tilted side face (light grey) or outside the NP (dark grey), i.e., inelastic electron tunneling cannot be modeled as a vertical electric dipole in these regions. Comparisons between theoretical and experimental results in (a) and (b) are only relevant in the unshaded regions. (d) Theoretical emission intensity $I$ in the substrate calculated using the three approaches described in the text (inset: NP shape used in the calculations). In (a), (b), and (d), results are shown as a function of the tip position along the median of the upper NP triangular face $(0$ corresponds to the center). Symbols with error bars are experimental data taken from Ref [32]; circles $(\odot)$ are the complete calculations including the full NP-tip geometry; diamonds $(\odot)$ are model calculations of the NP excited by the point dipole with no tip (i.e., Green's function of the NP); solid dots and line (-•-) are model calculations based on $E_{z}$ maps of plasmonic eigenfields.

less memory and time-consuming than those of (i) and (ii). While being less exact, approach (iii) has the advantage of retrieving all the data from a single calculation of the plasmonic eigenfields, while (i) and (ii) require calculations to be performed for each tip position. In (i) and (ii), the polarization density integrated over the NP or/and the NP and the tip volume defines the tip excitation position-dependent induced dipole $\mathbf{P}$, its tilt angle $\Theta$ with respect to the tip axis, and intensity of emitted radiation $I(\omega) \sim \omega^{4}|\mathbf{P}|^{2}$. Since in our calculations the $E_{z}$ maps of plasmonic fields are not normalized, applying approach (iii) implies that the $P_{\|} / P_{z}$ ratio be normalized for one tip position using, e.g., one point in (ii), and all the other points are then retrieved, for both $\Theta$ and $I$, from this single normalization step.

Figure 4 compares the results of the three theoretical approaches and experimental data taken from Ref. [32]. The effective dipole orientation $\Theta$ and the intensity of emitted radiation $I$ are shown as a function of the tip position along the median of the upper NP triangular face ( 0 corresponds to the center of the triangle). In our experiments, statistics does not allow to perform the radiation frequency resolved measurements. Therefore the intensity and the dipole orientation represent the values averaged over the radiated spectrum. To compare with experiment, the frequency resolved theoretical results have been integrated over the experimental energy window using a weighting function given by the $\omega$-dependent detection efficiency and the IET power spectrum $\left(\propto e U_{b}-\hbar \omega\right.$, for $\hbar \omega \leqslant e U_{b} ; 0$ otherwise [53]).

We observe in Fig. 4(a) that the theoretical results obtained for the complete NP-tip system [approach (i)] closely reproduce the experimentally determined dependence of $\Theta$, the effective dipole orientation, on the tip excitation position. Integration of the calculated polarization density of the NP-tip system over the gold NP and the W tip separately shows that the polarization of the $\mathrm{W}$ tip contributes about $30 \%$ to the vertical $P_{z}$ dipole of the total system, but has little effect on the horizontal polarization $P_{\|}$(less than 10\%). This result holds for all studied tip positions on the upper NP face. Thus the light emission from the STM junction between nonplasmonic (here W) tip and plasmonic (here $\mathrm{Au}$ ) NP is dominated by the polarization induced in the NP, which fully justifies the simplifying assumptions made in this work.

As follows from Fig. 4(b), the models (ii) and (iii), which neglect the presence of the STM tip, reproduce the experimentally measured effective dipole angle $\Theta$ on a semiquantitative level, correctly capturing all the main trends. A slightly larger tilt angle of the "effective dipole" is obtained in these simplified treatments because the tip polarization is neglected 
effectively reducing the $P_{z}$ component of the effective dipole. Remarkably, the calculation of the effective dipole angle $\Theta$ using (ii) the NP Green's function, and (iii) the simple model based on the $E_{z}$ maps of the plasmonic NP fields agree very well. This confirms the validity of the $E_{z}$ normalization and the utility of this simple approach.

Figure 4(d) shows the theoretical variation of the intensity of emitted light $I$ versus tip position, as calculated using the three approaches discussed above. Again, the model (ii) based on the calculation of the point dipole excitation of the NP (Green's function of the NP with no tip) and the (iii) simple model using $E_{z}$ maps of plasmonic NP fields are in relative agreement with each other. As well, both models reproduce the (i) complete calculation of the NPtip system on a semiquantitative level, correctly capturing all the main trends. An intensity minimum is found at the center of the upper (triangular) NP face and first and secondary maxima are found close to its vertex and basis, respectively.

The shaded areas in Figs. 4(a) and 4(b) indicate the positions where the tip is outside the upper NP face (light grey) and outside the NP (dark grey) so that experimental and calculated data cannot be compared because of boundary effects. First, theory assumes right-angled side faces so that the field structure at the NP boundaries is not reproduced. Second, within these areas the tunneling current and associated transition dipole are not aligned along the $\mathbf{z}$ axis. The difference between the complete and approximate theoretical treatments as appears in Fig. 4(d) close to the edges of the NP face can be also related to boundary effects. Indeed, despite the small curvature of the tip, in these regions the axial symmetry of the near field distribution below the tip apex should be strongly perturbed. The axial orientation assumption of the effective dipole does not hold in this case (the approximation $\mathbb{S} \rightarrow S$ is not valid).

We close this section with a remark about possible quantum and nonlocal effects. For the 1-nm wide junction, the effects due to the nonlocal screening might affect the electromagnetic response of the NP-tip system [54]. In the narrow plasmonic gaps, nonlocality mainly results in the weakening of the near fields and decreased plasmonic coupling [55]. In the present system, we expect a possible change of the amplitude of the effective dipole $\tilde{\mathbf{p}}$. This should not affect the tip position dependence of the light emission pattern. In addition, the nonlocal effects might modify the configuration of the induced fields close to the vertices of the NP face. However, the present approach does not apply to these regions as discussed above.

\section{CONCLUSIONS}

In conclusion, the use of a nonplasmonic tip allows flexible and predictable control of the STM-induced emission of light from a plasmonic NP on a transparent substrate. The intricate NP-tip system may be substituted by a much simpler model system where the plasmonic NP is excited by a point dipole and the tip is not explicitly included in the calculations. Our results greatly simplify both the understanding and prediction of the light emission properties of the system, since they are solely determined by the plasmonic modes of the NP. Since the local density of photonic states (photonic LDOS) is related to the Green's function [38], IET in the STM tunnel junction between a nonplasmonic tip and a plasmonic NP is a local probe of the photonic LDOS, similarly to cathodoluminiscence and scanning near field optical microscopy [35,38,56,57] (see also a related discussion in Ref. [58]). Though discussed here for a small particle and thus restricted to the dipolar modes, our approach applies to the general case of high-order multipolar excitations existing in larger particles. Spectral, spatial, and angular distributions, polarization and radiative rates are thus expected to be controllable using all the well-established strategies in nanophotonics to engineer the photonic LDOS. Our approach provides a simple means to optimize the design of electrically driven nanosources of light in view of their integration with microelectronics.

\section{ACKNOWLEDGMENTS}

A.G.B. and T.V.T. are indebted to A.K. Kazansky for many stimulating discussions. A.G.B. gratefully acknowledges hospitality of the Donostia International Physics Center. This work is supported by the Conseil Régional, Ile-de-France in the framework of DIM Nano-K and by "Investissements d' Avenir" LabEx NanoSaclay (ANR-10-LABX-0035-NanoSaclay).
[1] Nanoelectronics for 2020 and Beyond, National Nanotechnology Initiative, NSTC Commitee on Technology, http://www.nano.gov/node/612 (2010).

[2] V. J. Sorger, R. F. Oulton, R.-M. Ma, and X. Zhang, MRS Bull. 37, 728 (2012).

[3] K. C. Y. Huang, M.-K. Seo, T. Sarmiento, Y. Huo, J. S. Harris, and M. L. Brongersma, Nat. Photon. 8, 244 (2014).

[4] K. Ding, Z. C. Liu, L. J. Yin, M. T. Hill, M. J. H. Marell, P. J. van Veldhoven, R. Nöetzel, and C. Z. Ning, Phys. Rev. B 85, 041301 (2012).

[5] J. Kern, R. Kullock, J. C. Prangsma, M. Emmerling, M. Kamp, and B. Hecht, Nat. Photon. 9, 582 (2015).
[6] M. Parzefall, P. Bharadwaj, A. Jain, T. Taniguchi, K. Watanabe, and L. Novotny, Nat. Nanotechnol. 10, 1058 (2015).

[7] D. W. Pohl, S. G. Rodrigo, and L. Novotny, Appl. Phys. Lett. 98, 023111 (2011).

[8] J. Lambe and S. L. McCarthy, Phys. Rev. Lett. 37, 923 (1976).

[9] R. W. Rendell, D. J. Scalapino, and B. Mühlschlegel, Phys. Rev. Lett. 41, 1746 (1978).

[10] P. Dawson, D. G. Walmsley, H. A. Quinn, and A. J. L. Ferguson, Phys. Rev. B 30, 3164 (1984).

[11] P. Johansson, R. Monreal, and P. Apell, Phys. Rev. B 42, 9210 (1990). 
[12] B. N. J. Persson and A. Baratoff, Phys. Rev. Lett. 68, 3224 (1992).

[13] J. H. Coombs, J. K. Gimzewski, B. Reihl, J. K. Sass, and R. R. Schlittler, J. Microsc. 152, 325 (1988).

[14] P. Johansson and R. Monreal, Z. Phys. B Condens. Matter. 84, 269 (1991).

[15] R. Berndt, J. K. Gimzewski, and P. Johansson, Phys. Rev. Lett. 67, 3796 (1991).

[16] Y. Uehara, Y. Kimura, S. Ushioda, and K. Takeuchi, Jap. J. App. Phys. 31, 2465 (1992).

[17] R. Berndt, J. K. Gimzewski, and P. Johansson, Phys. Rev. Lett. 71, 3493 (1993).

[18] P. Johansson, Phys. Rev. B 58, 10823 (1998).

[19] F. Silly, A. O. Gusev, A. Taleb, F. Charra, and M.-P. Pileni, Phys. Rev. Lett. 84, 5840 (2000).

[20] N. Nilius, N. Ernst, and H.-J. Freund, Phys. Rev. Lett. 84, 3994 (2000).

[21] F. Silly, A. O. Gusev, A. Taleb, M.-P. Pileni, and F. Charra, Mater. Sci. Eng. C 19, 193 (2002).

[22] N. Nilius, N. Ernst, and H.-J. Freund, Phys. Rev. B 65, 115421 (2002).

[23] M. G. Boyle, J. Mitra, and P. Dawson, Appl. Phys. Lett. 94, 233118 (2009).

[24] L. Douillard and F. Charra, J. Phys. D: Appl. Phys. 44, 464002 (2011).

[25] P. Myrach, N. Nilius, and H.-J. Freund, Phys. Rev. B 83, 035416 (2011).

[26] F. Rossel, M. Pivetta, and W.-D. Schneider, Surf. Sci. Rep. 65, 129 (2010).

[27] J. Aizpurua, G. Hoffmann, S. P. Apell, and R. Berndt, Phys. Rev. Lett. 89, 156803 (2002).

[28] J. Aizpurua, S. P. Apell, and R. Berndt, Phys. Rev. B 62, 2065 (2000).

[29] G. Hoffmann, J. Aizpurua, P. Apell, and R. Berndt, Surf. Sci. 482-485, 1159 (2001).

[30] P. André, F. Charra, and M. P. Pileni, J. Appl. Phys. 91, 3028 (2002).

[31] A. Cvitkovic, N. Ocelic, J. Aizpurua, R. Guckenberger, and R. Hillenbrand, Phys. Rev. Lett. 97, 060801 (2006).

[32] E. Le Moal, S. Marguet, B. Rogez, S. Mukherjee, P. Dos Santos, E. Boer-Duchemin, G. Comtet, and G. Dujardin, Nano Lett. 13, 4198 (2013).

[33] T. H. Taminiau, F. D. Stefani, F. B. Segerink, and N. F. van Hulst, Nat. Photon. 2, 234 (2008).

[34] F. J. García de Abajo, Rev. Mod. Phys. 82, 209 (2010).

[35] T. Coenen, F. B. Arango, A. F. Koenderink, and A. Polman, Nat. Comm. 5, 3250 (2014).
[36] G. Colas des Francs, C. Girard, and A. Dereux, J. Chem. Phys. 117, 4659 (2002).

[37] C. Sauvan, J. P. Hugonin, I. S. Maksymov, and P. Lalanne, Phys. Rev. Lett. 110, 237401 (2013).

[38] R. Carminati, A. Cazé, D. Cao, F. Peragut, V. Krachmalnicoff, R. Pierrat, and Y. De Wilde, Surf. Sci. Rep. 70, 1 (2015).

[39] T. Wang, E. Boer-Duchemin, Y. Zhang, G. Comtet, and G. Dujardin, Nanotechnol. 22, 175201 (2011).

[40] A. G. Borisov and S. V. Shabanov, J. Comput. Phys. 209, 643 (2005).

[41] P. B. Johnson and R. W. Christy, Phys. Rev. B 6, 4370 (1972).

[42] C. Awada, T. Popescu, L. Douillard, F. Charra, A. Perron, H. Yockell-Lelièvre, A.-L. Baudrion, P.-M. Adam, and R. Bachelot, J. Phys. Chem. C 116, 14591 (2012).

[43] A. Losquin, L. F. Zagonel, V. Myroshnychenko, B. RodríguezGonzález, M. Tencé, L. Scarabelli, J. Förstner, L. M. LizMarzán, F. J. García de Abajo, O. Stéphan, and M. Kociak, Nano Lett. 15, 1229 (2015).

[44] S. Viarbitskaya, A. Teulle, R. Marty, J. Sharma, C. Girard, A. Arbouet, and E. Dujardin, Nat. Mater. 12, 426 (2013).

[45] L. Novotny and B. Hecht, Principles of Nano-Optics (Cambridge University Press, Cambridge, 2012).

[46] C. Grosse, M. Etzkorn, K. Kuhnke, S. Loth, and K. Kern, Appl. Phys. Lett. 103, 183108 (2013).

[47] K. Kaasbjerg and A. Nitzan, Phys. Rev. Lett. 114, 126803 (2015).

[48] C. Girard and A. Dereux, Rep. Prog. Phys. 59, 657 (1996).

[49] J.-J. Greffet and R. Carminati, Prog. Surf. Sci. 56, 133 (1997).

[50] L. Novotny and N. van Hulst, Nat. Photon. 5, 83 (2011).

[51] C. Neacsu, G. Steudle, and M. Raschke, Appl. Phys. B 80, 295 (2005).

[52] N. Behr and M. B. Raschke, J. Phys. Chem. C 112, 3766 (2008).

[53] D. Hone, B. Mühlschlegel, and D. J. Scalapino, Appl. Phys. Lett. 33, 203 (1978).

[54] R. Esteban, A. Zugarramurdi, P. Zhang, P. Nordlander, F. J. García-Vidal, A. G. Borisov, and J. Aizpurua, Faraday Discuss. 178, 151 (2015).

[55] S. Raza, S. I. Bozhevolnyi, M. Wubs, and N. A. Mortensen, J. Phys. Condens. Matter 27, 183204 (2015).

[56] F. J. García de Abajo and M. Kociak, Phys. Rev. Lett. 100, 106804 (2008).

[57] K. Joulain, R. Carminati, J.-P. Mulet, and J.-J. Greffet, Phys. Rev. B 68, 245405 (2003).

[58] A. García-Etxarri, I. Romero, F. J. García de Abajo, R. Hillenbrand, and J. Aizpurua, Phys. Rev. B 79, 125439 (2009). 